\title{
Evaporation sampled by stationary molecular dynamics simulation
}

\author{
Cite as: J. Chem. Phys. 151, 044704 (2019); doi: 10.1063/1.5111759 \\ Submitted: 29 May 2019 - Accepted: 5 July 2019 • \\ Published Online: 29 July 2019
}

Matthias Heinen and Jadran Vrabec

\begin{abstract}
AFFILIATIONS
Thermodynamik und Thermische Verfahrenstechnik, Technische Universität Berlin, Ernst-Reuter-Platz 1 , 10587 Berlin, Germany
\end{abstract}

a) Electronic mail: vrabec@tu-berlin.de

\begin{abstract}
A nonequilibrium method is developed to sample evaporation of a liquid across a planar interface in a stationary scenario by molecular dynamics. The method does not rely on particle insertions which are challenging when they are used to maintain mass conservation. Its algorithm has a low complexity and is well suited for massively parallel simulations that may yield results with an excellent statistical accuracy. Spatially resolved classical profiles, e.g., for temperature, density, and force, are sampled with a high resolution for a varying hydrodynamic velocity of the evaporation flow. Relatively large systems are simulated, allowing for a detailed study of velocity distribution functions. Varying the hydrodynamic velocity from zero to the speed of sound, it is found that the evaporation flux increases asymptotically, reaching about $90 \%$ of its maximum value when the hydrodynamic velocity is about half of its maximum value. A deviation from the Maxwell distribution is identified for the transversal particle velocity near the interface which selectively hinders the migration of individual particles from liquid to vapor with its potential well, allowing only the faster ones to escape. The vapor region in the vicinity of the interface exhibits a spread between the transversal and longitudinal temperature, but equipartition is reattained through particle interactions such that Maxwell distributions are found at a certain distance from the interface. A detailed discussion of the atomistic mechanisms during evaporation is provided, facilitating understanding of this ubiquitous process.
\end{abstract}

Published under license by AIP Publishing. https://doi.org/10.1063/1.5111759

\section{INTRODUCTION}

The evaporation of condensed matter is an elementary process encountered in many natural phenomena and is of great importance for technical applications. Its scientific exploration began with the experiments of Hertz ${ }^{1}$ in 1882. Also based on laboratory work, Knudsen ${ }^{2}$ introduced two empirical parameters, i.e., the evaporation and condensation coefficients, yielding the Hertz-Knudsen relation in its basic form. The Hertz-Knudsen relation and its modifications ${ }^{3,4}$ are widespread, being frequently used to correlate evaporation data, and serve as a basis for describing evaporation and condensation processes with kinetic theory.

Molecular dynamics (MD) simulation rests on a sound physical basis and has the undisputed strength to resolve the interface between the condensed phase and the vapor phase on the atomistic scale. Density profiles obtained from classical binning schemes uncover the transition from liquid to vapor density with an extent of a few particle diameters in both vapor-liquid equilibrium (VLE) and evaporation simulations. ${ }^{6}$ One of the earliest works on stationary evaporation across a vapor-liquid interface was published by Lotfi et al. ${ }^{7,8}$ Their results showed the temperature spread between the longitudinal and transversal temperature evolving at the interface, which was later confirmed by other $\mathrm{MD}^{9,10}$ and kinetic theory studies. ${ }^{11,12}$ This temperature spread characterizes local thermodynamic nonequilibrium in the vicinity of the interface as a consequence of the discontinuity of the velocity distribution function, deviating from the Maxwell distribution, due to the migration of particles across the potential well exerted by the interface.

Considering an evaporating two-phase system, the vapor phase at a sufficient distance from the interface, designated as the Euler regime, is in local thermodynamic equilibrium with itself and hence can be treated with continuum methods. ${ }^{13}$ Particles emitted from the interface impede local equilibrium in the adjacent Knudsen layer. While they propagate toward the Euler regime and interact on their way with other particles, they reattain the Maxwell velocity distribution at some distance from the interface, marking the 
boundary between the Knudsen layer and the Euler regime. ${ }^{14,15}$ The temperature spread is one characteristic of the Knudsen layer so that its extent can practically be determined by the plane where the temperature spread vanishes.

Investigations of evaporation processes have expanded to more complex systems, like droplet evaporation into inert gas ${ }^{16}$ and suspensions with nanoparticles, ${ }^{17}$ but recent reviews show that there is still a need for the clarification of basic questions. Persad and Ward ${ }^{3}$ examined sources of error in the Hertz-Knudsen relation. Consideration of a wide range of evaporation experiments with water and ethanol led to the conclusion that the Hertz-Knudsen relation is incomplete, missing a physical concept for coupling liquid and vapor phases during evaporation, since values for the evaporation and condensation coefficient have been found to span three orders of magnitude. Frezzotti and Barbante ${ }^{18}$ concluded in their review of kinetic aspects of nonequilibrium vapor-liquid flows that a complete description of the Knudsen layer structure with kinetic theory cannot be achieved without reliable kinetic boundary conditions to couple vapor and liquid phases. Therefore, standard models should be revised to possibly adapt suggested changes, like energy dependent condensation coefficients ${ }^{19-23}$ or the anisotropic shape of the distribution function of particles emitted from the liquid phase, as revealed by MD simulations. They are convinced that additional efforts have to be made to obtain a unified view of kinetic boundary conditions. One step to achieve this should be to validate the models through realistic flow simulations by comparison with experiments.

From our standpoint, this is a promising route to go. Therefore, we propose to extend the work of Meland et al. ${ }^{24}$ by performing MD simulations with sufficiently large vapor volumes covering the entire Knudsen layer up to the Euler regime. Data from such simulations can reveal whether kinetic boundary conditions, employed, e.g., in Direct Simulation Monte Carlo (DSMC) simulations, ${ }^{12}$ are capable of reproducing the structure of the Knudsen layer. Moreover, these data can serve as a benchmark for Computational Fluid Dynamics (CFD) simulations, augmented by derived jump formulas, ${ }^{25}$ to describe the coupling between the liquid and vapor phases. Finally, the results may be compared to experimental data.

A comparatively large extent of the vapor phase $L_{v}$ implies a longer communication distance between the interface and the control volume $\mathrm{CV}_{v}$ at the system boundary. Therefore, the propagation of a disturbance, generated by imposing a boundary condition that brings the system out of global equilibrium, takes longer until it reaches the interface. As a consequence, the simulation time until stationary conditions are achieved is increased too. Hence, a truly stationary method should be used for such investigations.

The conventional approach to satisfy mass conservation in stationary MD simulations ${ }^{8,24}$ is to remove particles that have reached a control volume in the vapor phase $\mathrm{CV}_{v}$ and insert them back into the liquid [cf. Fig. 1(a)]. Particles are usually inserted into a certain volume of the liquid that is at a sufficient distance from the interface to avoid perturbations of the evaporation process itself. One approach is to search for spatial positions in the liquid where the potential energy of an inserted particle is low. The USHER algorithm proposed by Delgado-Buscalioni and Coveney ${ }^{26}$ was designed to find such positions for particle insertions also in dense liquids. Since the number of appropriate positions at a certain time instance is small, it is difficult to find them with a high frequency in scenarios where

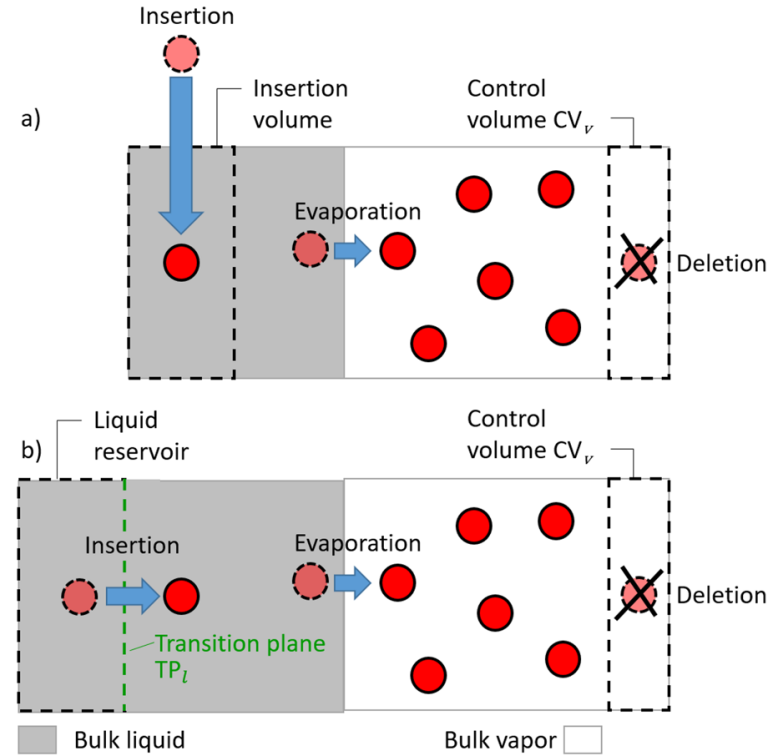

FIG. 1. Comparison between (a) the conventional insertion method and (b) the present approach.

the evaporation rate is high. Facing this issue by reducing the potential energy threshold for the acceptance of a particle position may lead to local instability of the liquid because too much energy per time may be added upon insertion. Similar problems apply for the gradual insertion method developed by Lotfi et al., where a particle was inserted as a point mass that stepwise attained its repulsive and attractive potential. A related method proposed by Meland et al. ${ }^{24}$ led to high local pressures that necessitated local thermostating of the insertion region. The splitting method of Zhang et al., ${ }^{27}$ where new particles were introduced by splitting selected particles into two that initially repel each other with a weak potential, was also tested for a comparatively high liquid temperature and hence a high evaporation rate. However, a drawback of this method is that test simulations need to be carried out before production runs to find a set of appropriate methodological parameters.

This work presents a new method to perform stationary evaporation with a specified hydrodynamic velocity of the vapor flow. In contrast to Meland et al., ${ }^{24}$ mainly a higher temperature of the liquid phase $T_{l}=0.8$ was investigated for the Lennard-Jones truncated and shifted fluid ${ }^{5}$ with a cutoff radius of 2.5 particle diameters $\sigma$, but a low temperature case $T_{l}=0.6$ was studied as well. Moreover, spatially resolved classical profiles, e.g., for temperature, density, and force, were sampled with a high resolution, distinguishing between the forward particle flux $j^{+}$and backward particle flux $j^{-}$, to deepen the understanding of the evaporation mechanisms on the atomistic scale.

\section{METHOD}

To achieve mass conservation and hence stationarity during evaporation, a reservoir adjacent to the liquid was introduced. Particles constituting this reservoir were in direct physical contact 
with those of the evaporating liquid through a transition plane [cf. Fig. 1(b)]. The present approach entirely avoids searching for appropriate insertion positions, is computationally cheap, and can straightforwardly be parallelized.

A reservoir to replenish the evaporating liquid was applied as shown in Fig. 2 (Multimedia view), where a liquid phase evaporated over a planar interface into an adjacent vapor phase. The reservoir contained a configuration of the homogeneous liquid phase, equilibrated at the same temperature and density, but the particles therein did not participate in individual thermal motion. Instead, they were collectively moved toward the evaporating liquid with an appropriate feed rate. Once particles crossed the transition plane $\mathrm{TP}_{l}$, they were allowed to move freely according to their interactions with their environment.

The dimensions of the reservoir were chosen such that its cross-sectional area $A_{x y}$ was the same as that of the simulation volume. Particles constituting the liquid were allowed to cross the transition plane $\mathrm{TP}_{l}$ into the reservoir without constraints. Therefore, the width of the reservoir should not be too small, which is important for high temperature liquids with a comparably low density. The feed rate $v_{f}$, with which reservoir particles were moved toward and across the transition plane $\mathrm{TP}_{l}$, had to satisfy mass conservation

$$
v_{f}=\frac{\Delta N}{A_{x y} \rho_{l} \Delta t},
$$

where $\Delta N$ is the number of particles deleted in the control volume $\mathrm{CV}_{v}$ per time step $\Delta t$ and $\rho_{l}$ is the density of the liquid. To replenish the liquid smoothly, reservoir particles were continuously moved toward and across the transition plane $\mathrm{TP}_{l}$ every time step over the distance $v_{f} \Delta t$. The feed rate $v_{f}$ was updated every 1000 th time step to suppress fluctuations and was averaged over the last 20 sampled values for additional smoothing. All physical properties are given below in a reduced form (cf. the supplementary material).

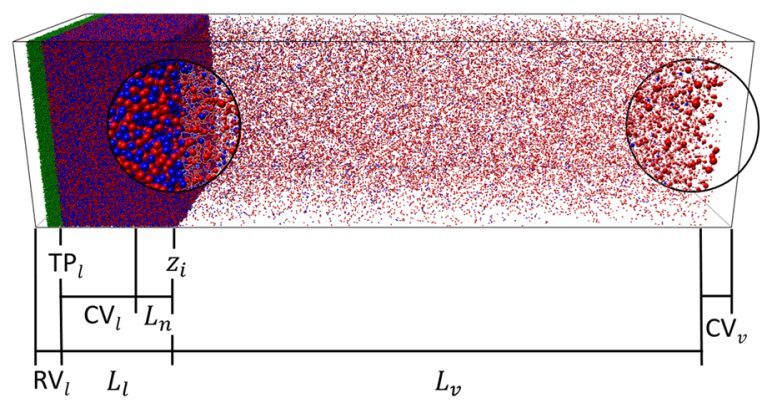

FIG. 2. Snapshot of a stationary simulation of evaporation into vacuum, visualized by MegaMol. ${ }^{28}$ The system consisted of a liquid and a vapor phase with an extent of $L_{i}$ and $L_{v}$, connected through a planar interface at the position $z_{i}=0$. To the left, the liquid was extended by a reservoir volume $\mathrm{RV}_{l}$, connected through the transition plane $\mathrm{TP}_{1}$. Interventions like thermostating were limited to the control volumes $\mathrm{CV}_{l}$ and $\mathrm{CV}_{v}$, leaving liquid and vapor phases in between to evolve naturally, which was the sampling region of interest. Particles constituting the reservoir, forward flux $j^{+}$, and backward flux $j^{-}$are colored green, red, and blue, respectively. Magnifying glasses (circles) are intended to support visualization. Multimedia view: https://doi.org/10.1063/1.5111759.1

\section{A. Evaporation into vacuum}

Vacuum was established by discarding all particles that have reached the control volume $\mathrm{CV}_{v}$ at a distance of $L_{v}$ from the interface [cf. Fig. 2 (Multimedia view)]. The liquid was replenished by employing the liquid reservoir such that the evaporation process was stationary. The starting point of every evaporation simulation was a VLE configuration. When particles that reached the control volume $\mathrm{CV}_{v}$ were discarded, the system initially reacted with a transient and instationary evaporation process, converging to stationary conditions after a certain time period. To understand the system's reaction to the vacuum boundary condition, the results of a VLE simulation at $T_{l}=0.8$ and those of an according vacuum evaporation simulation are compared by means of spatially resolved quantities that are depicted in Fig. 3. For orientation, the force profile $\hat{F}_{z}$ in Fig. 3(a) should be considered first. It shows the normalized mean force component in the $z$ direction,

$$
\hat{F}_{z}=\frac{F_{z}}{-\min \left(F_{z}\right)},
$$

defining the interface plane at $z_{i}=z\left(\hat{F}_{z}=-1\right)$ and the interface temperature $T_{i}=T\left(z_{i}\right)$, as proposed in Ref. 6 . It should be noted that the interface exerts a significant potential well that particles have to overcome when they migrate from one phase to the other.

The axis of the spatial coordinate $z$ was chosen such that its origin coincides with the interface position $z=z_{i}=0$ so that the density profile of a VLE simulation exhibits a typical transition from saturated liquid $\rho_{l}$ to saturated vapor $\rho_{v}$ there [cf. Fig. 3(c)]. It is well known ${ }^{5}$ that this transition has an extent of a few particle diameters $\sigma$; since the coordinate axis in Fig. 3 covers a range of several hundred particle diameters, the transition appears sharp in this illustration.

The density $\rho$ can be decomposed into partial densities,

$$
\rho=\rho^{+}+\rho^{-},
$$

where $\rho^{+}$and $\rho^{-}$consider only the particles constituting either the forward flux $j^{+}$or the backward flux $j^{-}$, respectively. As they should, VLE simulation data show perfectly overlapping $\rho^{+}$and $\rho^{-}$profiles, i.e., the number of particles moving forward or backward was on average balanced. The particle flux $j$ is given by

$$
j=\rho \bar{v}_{z},
$$

where the hydrodynamic velocity $\bar{v}_{z}=1 / N \sum_{j=1}^{N} v_{z, j}$ was averaged over the $N$ particle collective in a bin of a classical binning scheme. In analogy to Eq. (4),

$$
j^{+}=\rho^{+} v_{z}^{+}, \quad j^{-}=\rho^{-} v_{z}^{-},
$$

where $v_{z}^{+}$and $v_{z}^{-}$are the average velocities of the particle collective constituting either the forward flux $j^{+}$or the backward flux $j^{-}$, respectively. The net flux $j$ is then simply the sum

$$
j=j^{+}+j^{-} .
$$

Equations (3) and (6) indicate that the hydrodynamic velocity $\bar{v}_{z}$ can be derived from the partial velocities $v_{z}^{+}$and $v_{z}^{-}$and partial densities $\rho^{+}$and $\rho^{-}$. VLE conditions imply a zero net flux $j=0$ and hence also a vanishing hydrodynamic velocity $\bar{v}_{z}=0$ so that $\rho^{+} v_{z}^{+}=-\rho^{-} v^{-}$. 

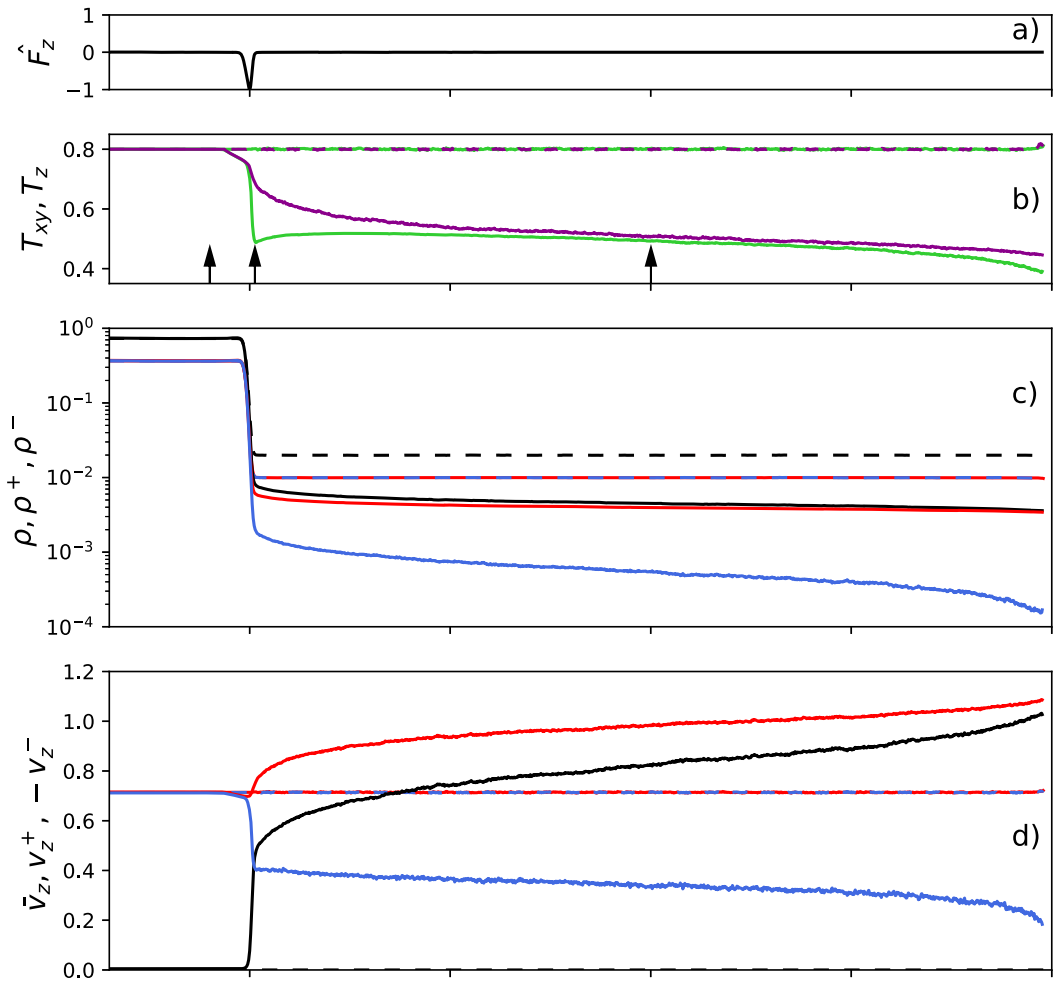

b)

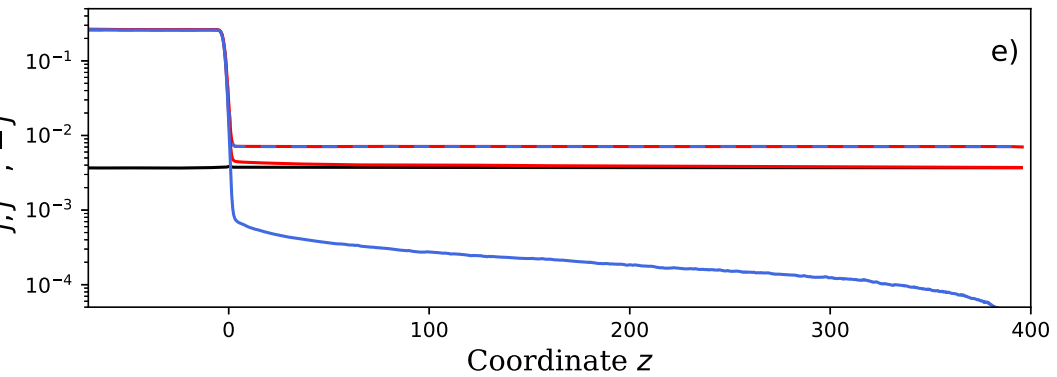

FIG. 3. Profiles obtained from VLE (dashed lines) and evaporation into vacuum simulations (solid lines) for $T_{1}=0.8$ and $L_{v}=400$ : (a) normalized force component $\hat{F}_{z}$; (b) longitudinal temperature $T_{x y}$ (purple) and transversal temperature $T_{z}$ (green), indicating positions $z=-10$, 2 , and 200 by arrows; (c) density $\rho$ (black) and partial densities $\rho^{+}$(red) and $\rho^{-}$(blue); (d) hydrodynamic velocity $\bar{v}_{z}$ (black) and partial velocities $v_{z}^{+}$(red) and $v_{z}^{-}$(blue); (e) net particle flux $j$ (black), forward flux $j^{+}$(red), and backward flux $j^{-}$(blue). Partial quantities $\rho^{+}, \rho^{-}$and $v_{z}^{+}, v_{z}^{-}$were sampled considering only particles constituting either the forward flux $j^{+}$or the backward flux $j^{-}$, respectively.

During VLE simulation, $\rho^{+}=\rho^{-}$and $v_{z}^{+}=-v_{z}^{-}$were indeed observed [cf. Figs. 3(c) and 3(d)]. Moreover, $v_{z}^{+}=-v_{z}^{-}=$const. applies because of thermal equilibrium throughout the system. Consequently, also overlapping profiles of $j^{+}$and $-j^{-}$were obtained [cf. Fig. 3(e)], and the shape of these profiles is the same as that of the density profiles $\rho^{+}$and $\rho^{-}$, indicating a larger particle flux in the liquid phase than in the vapor phase according to their density ratio.

The vacuum boundary condition imposed by deleting all particles in the control volume $\mathrm{CV}_{v}$ forces the partial density of the backward flux at the position $L_{v}$ to be zero, i.e., $\rho^{-}\left(z=L_{v}\right) \equiv 0$. Starting from VLE conditions, the perturbation of the equilibrium after imposing the vacuum propagated toward the interface like a rarefaction wave [cf. the supplementary material]. Once stationary conditions were attained, the partial density $\rho^{-}$at the interface was reduced by about one order of magnitude. Toward the vacuum, $\rho^{-}$ decreased by almost another order of magnitude, and in the vicinity of the vacuum, a progressively steeper descent to zero was observed.
This decrease in $\rho^{-}$indicates the rarefaction of the backward flux $j^{-}$. A further decrease in $j^{-}$was caused by a spread between the partial velocities $v_{z}^{+}>-v_{z}^{-}$. Considering individual particles of the vapor phase under VLE conditions, the probability of interacting with another particle being part of the forward flux $j^{+}$or the backward flux $j^{-}$is equal. Under evaporation conditions, however, the majority of interaction partners was part of the forward flux $j^{+}$. As a consequence, the distribution of the velocity component $v_{z}$ was shifted toward positive values. Hence, compared to VLE conditions, an increased average velocity of the forward flux $v_{z}^{+}$and a decreased average velocity of the backward flux $v_{z}^{-}$were obtained, leading to a further decrease in $j^{-}$as indicated above. This situation entailed a hydrodynamic velocity $\bar{v}_{z}$ in the vapor phase, showing a steep rise in the vicinity of the interface, turning into a much less pronounced further ascent toward the vacuum. Consequently, a flux $j>0$ evolved that was constant throughout the system due to stationary evaporation. 
These changes also had consequences for the energy balance at the interface. Under VLE conditions, the energy transported away from the interface by particles of the forward flux $j^{+}$was returned by particles of the backward flux $j^{-}$. Under evaporation conditions, however, the number of particles arriving at the interface and their energy were decreased so that the interface temperature $T_{i}$ had to drop down to satisfy the energy conservation principle. This temperature drop counters the mechanisms that bring the energy balance at the interface out of equilibrium by different effects. Due to lower particle velocities and a higher surface tension associated with a lower interface temperature, the evaporation flux $j$ decreases. ${ }^{6}$ Simultaneously, an increased temperature gradient $T_{l}-T_{i}$ and a reduced heat conduction resistance due to an increased density of the colder liquid raise the heat supply of the interface from the liquid side. The interface temperature $T_{i}$ falls until the energy balance is in equilibrium and attains a constant value under stationary evaporation conditions. The magnitude of the temperature drop $T_{l}$ - $T_{i}$ depends on the extent $L_{n}$ of the nonthermostated liquid.

Figure 3(b) shows the temperature profiles sampled for the relevant spatial directions $T_{x y}=\left(T_{x}+T_{y}\right) / 2$ and $T_{z}$, corresponding to a longitudinal and a transversal temperature, as designated by Frezzotti et al. ${ }^{11}$ In the vapor phase, the temperature profiles diverge, where $T_{x y}>T_{z}$. This issue was observed already by many authors. $8,10,11,29$

To obtain a more detailed insight, the spatially resolved distribution of particle velocity $v_{z}$ is shown in Fig. 4 for three different $z$ positions as indicated in Fig. 3(b). The first one was located inside the thermostated liquid bulk, the second one was in the vapor close to the interface position $z_{i}$ where the largest temperature spread was observed, and the third one was half way between the interface and the vacuum, i.e., $z=-10,2$, and 200 for $L_{v}=400$. When hydrodynamic motion is present, the probability density function $\operatorname{pdf}\left(v_{z}\right)$ of
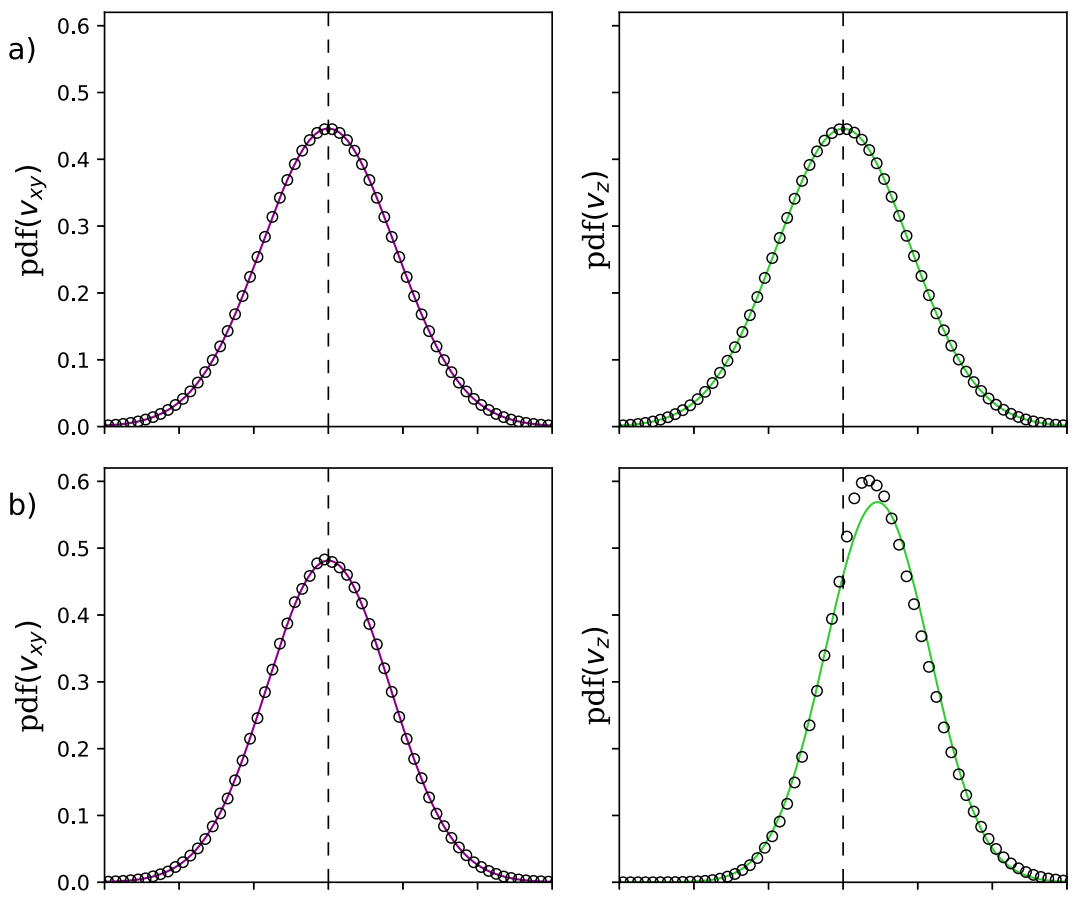

FIG. 4. Velocity distributions $\operatorname{pdf}\left(v_{x y}\right)$ and $\operatorname{pdf}\left(v_{z}\right)$ sampled at three different positions: (a) $z=-20$, (b) $z=2$, and (c) $z=200$, as indicated in Fig. $3($ b) The simulation results (circles) are contrasted to Maxwell distributions (solid line) obtained from Eqs. (7) and (8), applying values for $T_{x y}, T_{z}$, and $\bar{v}_{z}$ from binning for distribution width and hydrodynamic velocity.
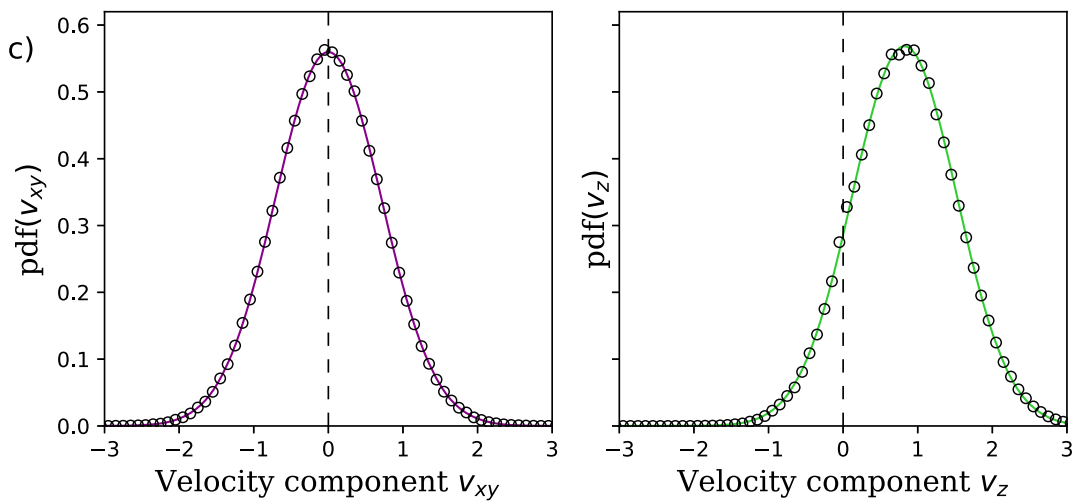
the particle velocity $v_{z}$ follows a normal distribution

$$
\operatorname{pdf}\left(v_{z}\right)=\frac{1}{\sqrt{2 \pi \sigma^{2}}} \exp \left(-\frac{\left(v_{z}-\bar{v}_{z}\right)^{2}}{2 \sigma^{2}}\right),
$$

where the expectation value is the hydrodynamic velocity $\bar{v}_{z}$ and the variance $\sigma^{2}$ determines the distribution width. Since there was only thermal motion in $x$ and $y$ directions in the present scenario, $\bar{v}_{x}=\bar{v}_{y}=0$. Consequently, $v_{x}$ and $v_{y}$ can be summarized to $v_{x y}$ and the according probability density function simplifies to

$$
\operatorname{pdf}\left(v_{x y}\right)=\frac{1}{\sqrt{2 \pi \sigma^{2}}} \exp \left(-\frac{v_{x y}^{2}}{2 \sigma^{2}}\right) .
$$

Figure 4 contrasts velocity distributions sampled by present simulations to $\operatorname{pdf}\left(v_{z}\right)$ and $\operatorname{pdf}\left(v_{x y}\right)$ obtained from Eqs. (7) and (8), applying values for

$$
\begin{aligned}
& T_{z}=\frac{m}{N k_{B}} \sum_{j=1}^{N}\left(v_{z, j}-\bar{v}_{z}\right)^{2}, \\
& T_{x y}=\frac{m}{2 N k_{B}} \sum_{j=1}^{N} v_{x, j}^{2}+v_{y, j}^{2},
\end{aligned}
$$

sampled by binning to calculate the distribution widths $\sigma^{2}=k_{B} T_{z} / m$ and $\sigma^{2}=k_{B} T_{x y} / m$, respectively, where $k_{B}$ is the Boltzmann constant and $m$ is the particle mass. It shows that the particle velocities $v_{z}$ and $v_{x y}$ in the bulk liquid are perfectly Maxwell distributed. However, at the position of the largest temperature spread, significant deviations from the Maxwell distribution were observed. The

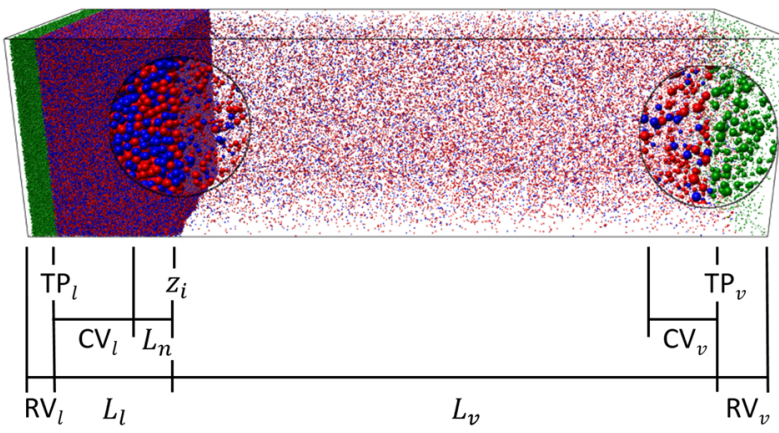

FIG. 5. Snapshot of a stationary simulation of evaporation, visualized by MegaMol. ${ }^{28}$ The system consisted of a liquid and a vapor phase with an extent of $L_{l}$ and $L_{v}$, connected through a planar interface at the position $z_{i}=0$. At both outer boundaries, the phases were extended by reservoir volumes $\mathrm{RV}_{\text {I }}$ and $\mathrm{RV}_{v}$, connected through transition planes $\mathrm{TP}_{/}$and $\mathrm{TP}_{v}$, respectively. Interventions like thermostating were limited to the control volumes $\mathrm{CV}_{l}$ and $\mathrm{CV}_{v}$, leaving liquid and vapor phases in between to evolve naturally, which was the sampling region of interest. Particles constituting the reservoirs, forward flux $j^{+}$, and backward flux $j^{-}$are colored green, red, and blue, respectively. Magnifying glasses (circles) are intended to support visualization. Multimedia view: https://doi.org/10.1063/1.5111759.2

sampled distribution of $v_{z}$ was not symmetric around the most probable velocity, which also did not coincide with the hydrodynamic velocity $\bar{v}_{z}$ but was shifted to slightly smaller values. For compensation, higher velocities were encountered more frequently than in the Maxwell distribution. This can be explained by the fact that those particles are more likely to escape from the potential well exerted by the interface.

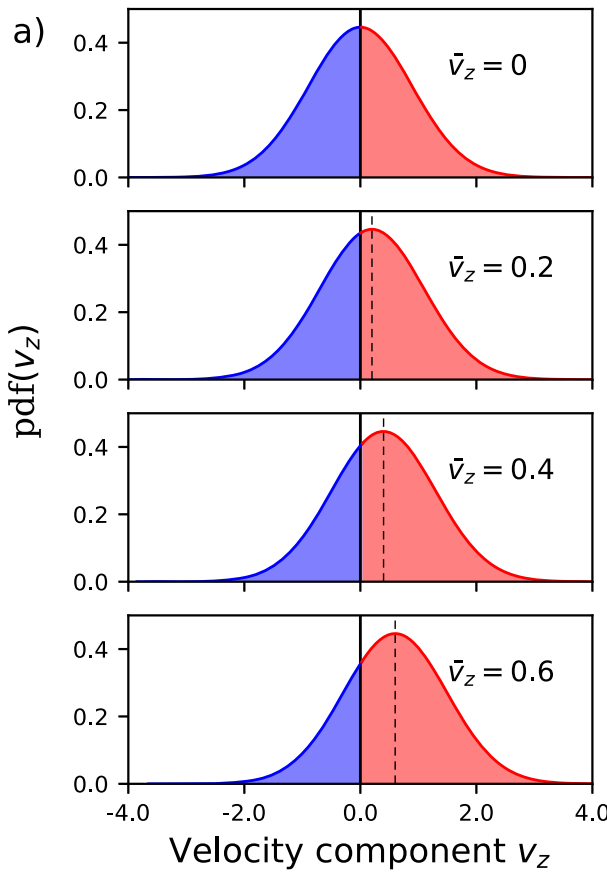

b)
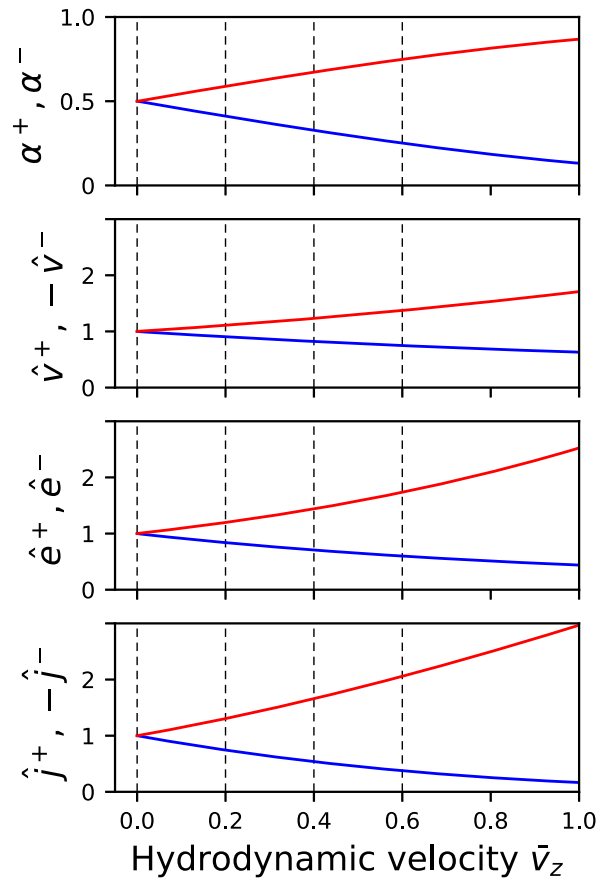

FIG. 6. (a) Shifted Maxwell distributions of gas flows with a temperature $T=0.8$ for varying hydrodynamic velocities $\bar{v}_{z}=0,0.2,0.4$, and 0.6. (b) Quantities of the forward and backward flux as a function of hydrodynamic velocity, where the dashed vertical lines indicate cases depicted in panel (a). 


\section{B. Evaporation with counterpressure}

Counterpressure during evaporation was established with a kinetic boundary condition by imposing a backward flux $j^{-}$across the vapor transition plane $\mathrm{TP}_{v}$ [cf. Fig. 5 (Multimedia view)]. On their way toward the interface, particles constituting the backward flux $j^{-}$interacted with those of the forward flux $j^{+}$so that some were scattered back before they reached the interface, leading to an energy redistribution between the opposing fluxes. This effect, together with the energy transport of particles to the interface, determined the system behavior. Interventions on the vapor side were limited in this work to backward flux particles in the control volume $\mathrm{CV}_{v}$ so that the forward flux $j^{+}$evolved naturally. This follows the lines of Ishiyama et al., ${ }^{30}$ who also generated the backward flux $j^{-}$by inserting particles on an insertion plane at a constant distance to the interface. The present approach differs only in terms of implementation. For convenience, the algorithm for replenishing the liquid was also used to generate the backward flux $j^{-}$of the vapor phase. Here, the vapor phase, equilibrated at the temperature $T_{l}$ of the liquid, served as a reservoir configuration. The continuous insertion of particles from the reservoir volume $\mathrm{RV}_{v}$ operated in the same way as described for the liquid side, except that the reservoir particle collective $\mathrm{RV}_{v}$ was moved in the opposite direction toward and across the transition plane $\mathrm{TP}_{v}$.

The basic idea was to impose a backward flux $j^{-}$with a Maxwell velocity distribution that is consistent with the response of the evaporating two-phase system, i.e., a propagating vapor phase. As an independent target property that governs the evaporation process for a given temperature of the liquid, the hydrodynamic velocity $\bar{v}_{z}$ was selected.

Hence, particles entering the simulation volume through the transition plane $\mathrm{TP}_{v}$ had to be assigned with velocities that adhere to the probability density functions $\operatorname{pdf}\left(v_{x y}\right)$ and $\operatorname{pdf}\left(v_{z}\right)$ according to the evolving temperature of the vapor phase while also taking its evolving density into account. Assigning $v_{x}$ and $v_{y}$ was straightforward since $\operatorname{pdf}\left(v_{x y}\right)$ depends only on the temperature due to $\bar{v}_{x}=\bar{v}_{y}=0$, whereas for assigning $v_{z}$, the hydrodynamic velocity $\bar{v}_{z}$ and its influence on $\operatorname{pdf}\left(v_{z}\right)$ had to be considered.

Figure 6(a) shows velocity distributions for the temperature $T=0.8$ and varying hydrodynamic velocities of $\bar{v}_{z}=0,0.2,0.4$, and 0.6. On that basis, several quantities of the forward flux $j^{+}$and backward flux $j^{-}$can be derived. If the density $\rho$ is known, the partial densities of the forward flux $j^{+}$and backward flux $j^{-}$particles are

$$
\rho^{+}=\alpha^{+} \rho, \quad \rho^{-}=\alpha^{-} \rho
$$

and are determined by

$$
\alpha^{+}=\int_{0}^{+\infty} \operatorname{pdf}\left(v_{z}\right) d v_{z}, \quad \alpha^{-}=\int_{-\infty}^{0} \operatorname{pdf}\left(v_{z}\right) d v_{z},
$$

since $\operatorname{pdf}\left(v_{z}\right)$ is normalized,

$$
\int_{-\infty}^{+\infty} \operatorname{pdf}\left(v_{z}\right) d v_{z}=1
$$

Figure 6(b) shows how the flux quantities depend on the hydrodynamic velocity

$$
\bar{v}_{z}=\int_{-\infty}^{+\infty} v_{z} \operatorname{pdf}\left(v_{z}\right) d v_{z}
$$

The partial velocities $v_{z}^{+}$and $v_{z}^{-}$are given by the first moment,

$$
v_{z}^{+}=\frac{1}{\alpha^{+}} \int_{0}^{+\infty} v_{z} \operatorname{pdf}\left(v_{z}\right) d v_{z}, \quad v_{z}^{-}=\frac{1}{\alpha^{-}} \int_{-\infty}^{0} v_{z} \operatorname{pdf}\left(v_{z}\right) d v_{z},
$$

while the partial kinetic energies $e^{+}$and $e^{-}$are given by the second moment,

$e^{+}=\frac{m}{2 \alpha^{+}} \int_{0}^{+\infty} v_{z}^{2} \operatorname{pdf}\left(v_{z}\right) d v_{z}, \quad e^{-}=\frac{m}{2 \alpha^{-}} \int_{-\infty}^{0} v_{z}^{2} \operatorname{pdf}\left(v_{z}\right) d v_{z}$.

Under VLE conditions, the hydrodynamic velocity vanishes $\bar{v}_{z}=0$ and hence $\operatorname{pdf}\left(v_{z}\right)$ is symmetric, yielding $\alpha^{+}=\alpha^{-}=1 / 2$ and $\rho^{+}=\rho^{-}$ $=\rho / 2$. Other flux quantities are then also balanced, i.e., $v_{z}^{+}=-v_{z}^{-}$, $e^{+}=e^{-}$, and $j^{+}=-j^{-}$. Under evaporation conditions, these properties diverge with increasing $\bar{v}_{z}$. The number of particles moving forward increases, whereas the number of particles moving backward decreases, indicated by $\alpha^{+}>\alpha^{-}$and $\rho^{+}>\rho^{-}$. The same applies for the other quantities $v_{z}^{+}>-v_{z}^{-}, e^{+}>e^{-}$, and $j^{+}>-j^{-}$.

Appropriate velocity vectors had to be assigned to the particles released into the simulation volume across $\mathrm{TP}_{v}$ to establish the backward flux $j^{-}$that is consistent with the specified target hydrodynamic velocity. For generating normal distributed random numbers according to Eq. (7), a standard algorithm ${ }^{31}$ was used. Numbers for

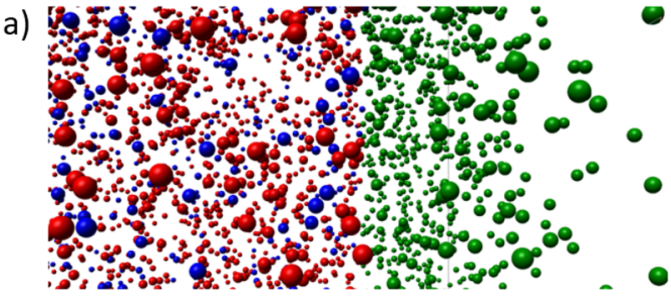

b)

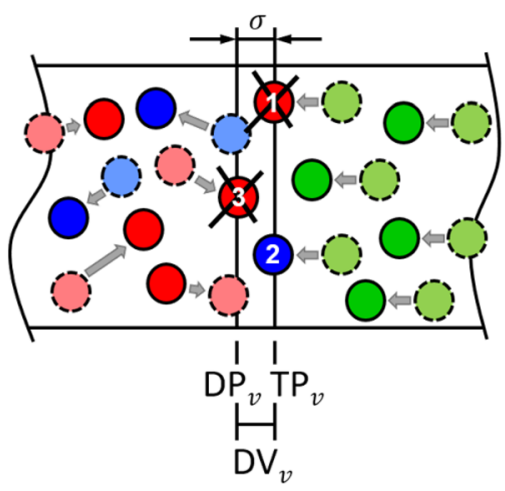

FIG. 7. (a) Snapshot of the region connecting the sampling volume and the vapor reservoir from a stationary evaporation simulation with counterpressure. (b) Schematic view of that region. A deletion volume $\mathrm{DV}_{v}$ was located between the deletion plane $\mathrm{DP}_{v}$ and the transition plane $\mathrm{TP}_{v}$ and had an extent of one particle diameter. Particles constituting the vapor reservoir, forward flux $j^{+}$, and backward flux $j^{-}$are colored green, red, and blue, respectively. Two time instances are shown in panel (b): dashed edges indicate the earlier, solid edges the later one. Coming from the vapor reservoir, particles 1 and 2 crossed the transition plane $T P_{v}$ to join either the forward $j^{+}$or backward flux $j^{-}$according to $\alpha^{+}$and $\alpha^{-}$. Particles of $j^{+}$were discarded in the deletion volume $\mathrm{DV}_{v}$. 
$v_{x}$ and $v_{y}$ were distributed around zero, whereas numbers for $v_{z}$ were distributed around the target hydrodynamic velocity $\bar{v}_{z}$, accepting only negative values $v_{z}<0$. Before the velocity vectors $\left(v_{x}, v_{y}, v_{z}\right)$ were assigned to particles of $j^{-}$released into the simulation volume, an offset correction to the target hydrodynamic velocity $\bar{v}_{z}$ was made and subsequent scaling of the kinetic energy $e^{-}$was conducted. Finally, the appropriate density of the backward flux had to be imposed that obeys $j^{-}=\rho^{-} v_{z}^{-}$. Therefore, particles constituting the vapor reservoir were continuously moved with a constant feed rate $v_{f}=v_{z}^{-}$toward and across the transition plane $\mathrm{TP}_{v}$ (cf. Fig. 7). After crossing the transition plane $\mathrm{TP}_{v}$, particles were either assigned to the forward flux $j^{+}$or the backward flux $j^{-}$, employing random numbers according to the fractions $\alpha^{+}$and $\alpha^{-}$, also leading to the partial densities $\rho^{+}$and $\rho^{-}$[cf. Eq. (11)]. Particles constituting the forward flux $j^{+}$were immediately discarded in the deletion volume $\mathrm{DV}_{v}$ (cf. Fig. 7). The deletion and transition planes $\mathrm{DP}_{v}$ and $\mathrm{TP}_{v}$ were arranged to be one particle diameter apart so that particles constituting $j^{-}$could interact repulsively with those of $j^{+}$only after they were released into the system.

Data for setting up stationary evaporation simulations with counterpressure were taken from VLE and vacuum evaporation simulations which are the two limiting cases. For a given temperature of the bulk liquid $T_{l}$, VLE data yield the maximum density of the vapor phase, i.e., the saturated density $\rho_{v}$, and the hydrodynamic velocity vanishes $\bar{v}_{z}=0$. A simulation into vacuum provided information about the minimum temperature and density as well as the maximum hydrodynamic velocity $\bar{v}_{z}$ of the vapor that can be reached which is close to the speed of sound. ${ }^{4}$ The minimum density is not zero. In the vicinity of the vacuum, $\rho^{-}=0$ indeed applies but a finite forward flux density $\rho^{+}>0$ remains.

To specify the hydrodynamic velocity $\bar{v}_{z}$ at that boundary, leaving all other properties to evolve naturally as a response of the
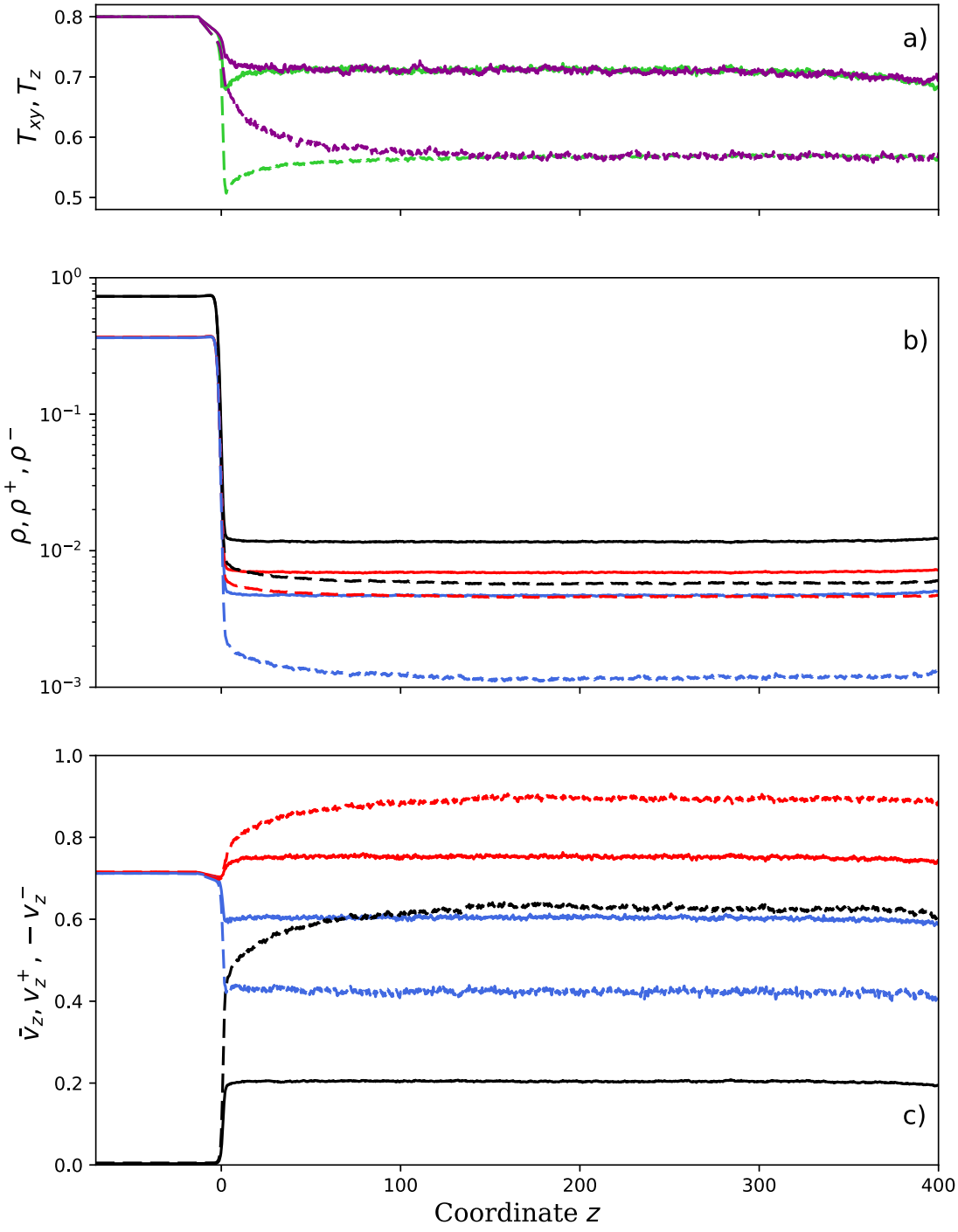

FIG. 8. Profiles obtained from evaporation simulations with $T_{I}=0.8, L_{v}=400$, and two different target hydrodynamic velocities $\bar{v}_{z}=0.2$ (solid lines) and 0.6 (dashed lines): (a) Iongitudinal temperature $T_{x y}$ (purple) and transversal temperature $T_{z}$ (green); (b) density $\rho$ (black) and partial densities $\rho^{+}$(red) and $\rho^{-}$(blue); (c) hydrodynamic velocity $\bar{v}_{z}$ (black) and partial velocities $v_{z}^{+}$(red) and $v_{z}^{-}$(blue). 
system, only the partial density of the backward flux was maintained to comply with the condition $\rho^{-}=\rho \alpha^{-} / \alpha^{+}$[cf. Eq. (11)]. Since the evolving partial density $\rho^{+}$of the forward flux is not known a priori, the number of particles released into the system was adjusted until the target hydrodynamic velocity $\bar{v}_{z}$ was attained. The velocity vectors assigned to those particles were generated according to the evolving temperature $T=\left(2 T_{x y}+T_{z}\right) / 3$ in the control volume $\mathrm{CV}_{v}$ and the specified hydrodynamic velocity $\bar{v}_{z}$ that determine the target velocity distributions [cf. Eqs. (7) and (8)].

\section{RESULTS AND DISCUSSION}

Figure 8 shows temperature, density, and hydrodynamic velocity profiles sampled during evaporation simulations with two different target hydrodynamic velocities $\bar{v}_{z}=0.2$ and 0.6 . As expected, beyond a certain distance from the interface toward the system boundary on the vapor side, all profiles show a flat course (zero gradient), indicating a uniformly propagating vapor phase. The attained hydrodynamic velocities of the vapor phase were in good agreement with the target values. Following the profiles from the liquid to the vapor boundary, a transition from the values at the interface toward constant values in the vapor phase was observed. This transition appears broader in the $\bar{v}_{z}=0.6$ case, indicating a larger Knudsen layer. This can be explained by the fact that the higher hydrodynamic velocity was achieved by imposing a lower density of the backward flux $\rho^{-}$, leading to fewer interactions between the particles of the opposing fluxes $j^{+}$and $j^{-}$. Consistent with Fig. 6, a larger ratio between the partial densities $\rho^{+} / \rho^{-}$and a larger spread between $v^{+}$and $-v^{-}$were found for $\bar{v}_{z}=0.6$. The combination of low density $\rho^{-}$and low velocity $-v^{-}$of the backward flux had the consequence that less energy was transported back to the interface, yielding a lower interface temperature $T_{i}$ and a more pronounced temperature
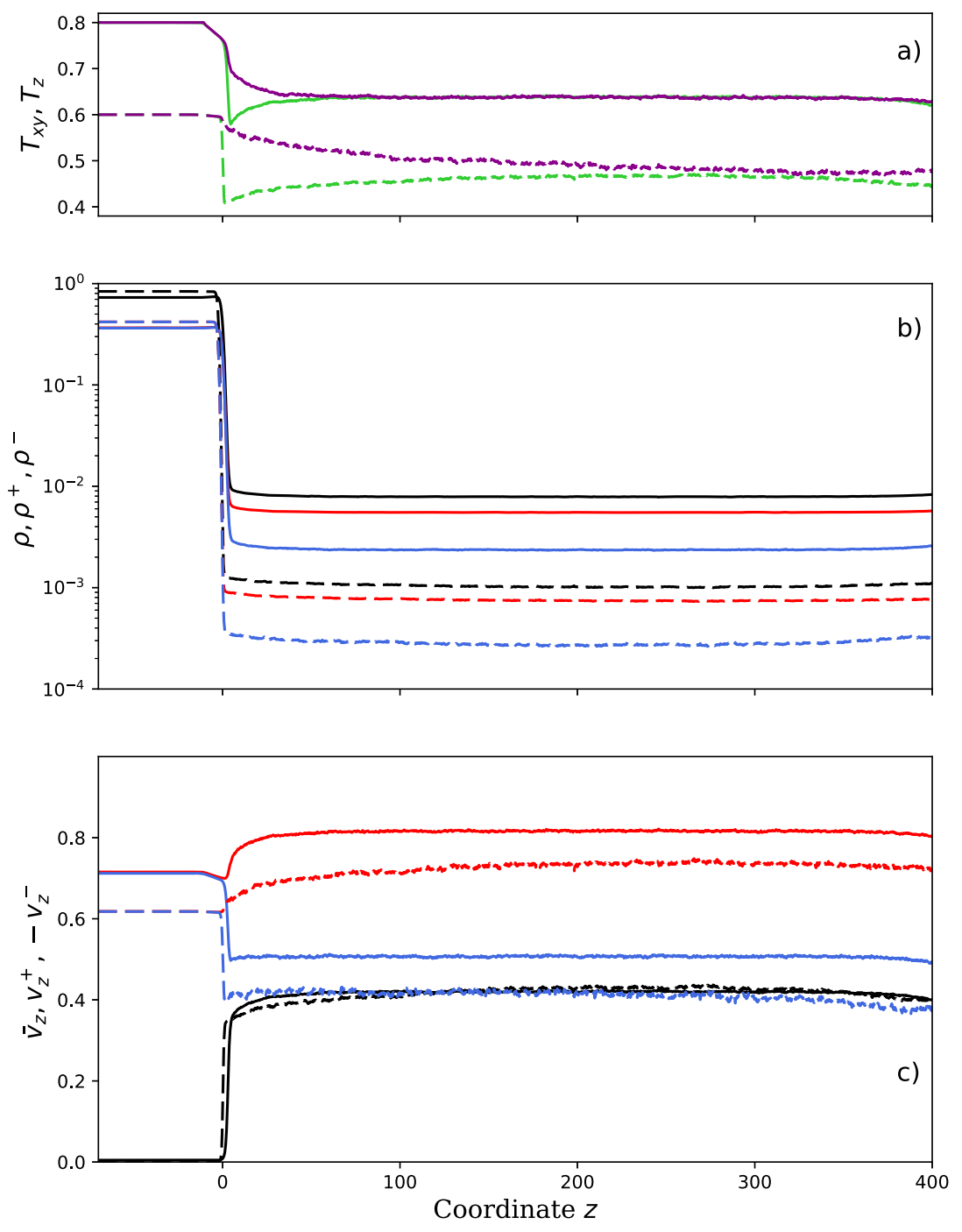

FIG. 9. Profiles obtained from evaporation simulations with $L_{v}=400, \bar{v}_{z}=0.4$ and two different liquid temperatures $T_{1}$ $=0.8$ (solid lines) and 0.6 (dashed lines): (a) longitudinal temperature $T_{x y}$ (purple) and transversal temperature $T_{z}$ (green); (b) density $\rho$ (black) and partial densities $\rho^{+}$(red) and $\rho^{-}$(blue); (c) hydrodynamic velocity $\bar{v}_{z}$ (black) and partial velocities $v_{z}^{+}$(red) and $v_{z}^{-}$(blue). 

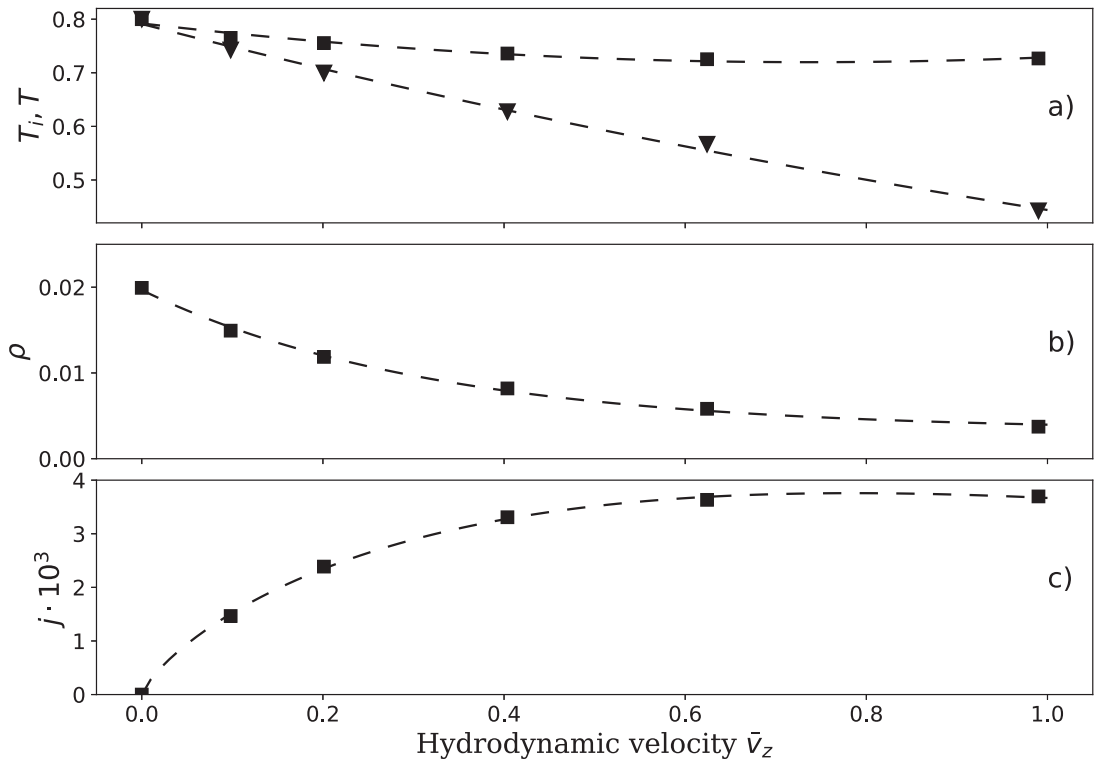

FIG. 10. (a) Interface temperature $T$ (squares) and vapor temperature close to the boundary $T$ (triangles). (b) Vapor density close to the boundary $\rho$. (c) Evaporation flux $j$. All properties are shown as a function of hydrodynamic velocity $\bar{v}_{z}$, where $\bar{v}_{z}=0$ corresponds to the VLE, $\bar{v}_{z}=0.1,0.2,0.4$, and 0.6 to evaporation with counterpressure, and $\bar{v}_{z} \approx 1$ to evaporation into vacuum. Dashed lines are guides to the eye. spread. Because of the lower interface temperature $T_{i}$, fewer particles escaped from the interface, leading to a lower density $\rho^{+}$of the forward flux. A further decrease in $\rho^{+}$was caused by the lower density $\rho^{-}$because fewer particles from the vapor side impinged on the interface and thus fewer particles were reflected to join the forward flux $j^{+}$.

The results of evaporation simulations with the same target hydrodynamic velocity $\bar{v}_{z}=0.4$ but different liquid temperatures $T_{l}=0.6$ and 0.8 are compared in Fig. 9. As expected, the density profiles for $T_{l}=0.6$ show a steeper transition from liquid to vapor, where the density of the latter is about one order of magnitude lower than that in the $T_{l}=0.8$ case. This low vapor density, implying a low partial density $\rho^{+}$, indicates a small forward flux $j^{+}$accompanied by a minor energy transport from the interface to the vapor phase. Consequently, a weak temperature gradient $T_{l}-T_{i}$ (not visible in Fig. 9) was sufficient to maintain the heat flux from the liquid to the interface that led to a constant interface temperature $T_{i}$ under stationary conditions. Moreover, the low vapor density and thus fewer particle interactions entailed a more pronounced temperature spread that persisted all the way up to the vapor boundary.

According to the sharper interface indicated by the density profile, also a steeper transition from the hydrodynamic velocity $\bar{v}_{z}$ of the liquid to that of the vapor phase was observed for the $T_{l}=0.6$ case. The partial velocity profiles $v^{+}$and $v^{-}$show almost the same courses but are shifted to lower values according to the reduced thermal motion in the liquid phase that caused a further decrease in the particle fluxes $j^{+}$and $j^{-}$.

Figure 10 summarizes the simulation results from VLE, over evaporation with counterpressure, to evaporation into vacuum, by depicting the most relevant quantities for $T_{l}=0.8$ and $L_{v}=400$ as a function of the target hydrodynamic velocity $\bar{v}_{z}$. As expected, higher hydrodynamic velocities $\bar{v}_{z}$ were associated with a lower vapor density $\rho$. The evaporation flux $j$ increased asymptotically with higher values of $\bar{v}_{z}$ to the maximum flux that was found in case of evaporation into vacuum. Interestingly, in case of $\bar{v}_{z}=0.4$, where $\approx 40 \%$ of the saturated vapor density $\rho_{v}$ was attained, already $\approx 90 \%$ of the maximum evaporation flux $j$ was present.

The interface temperature $T_{i}$ decreased asymptotically with higher values of $\bar{v}_{z}$ toward its minimum value, whereas the temperature of the vapor phase close to the boundary $T$ decreased almost linearly. From $\bar{v}_{z}=0.6$ to $\bar{v}_{z} \approx 1$, no further decrease in $T_{i}$ was observed, whereas $T$ was decreased by $\approx 20 \%$. This issue can be explained when reading Fig. 10 from right to left: A higher vapor density $\rho$ was achieved by imposing a higher partial density $\rho^{-}$of the backward flux $j^{-}$. As a consequence, more interactions between the particles of the opposing fluxes led to a decreased hydrodynamic velocity $\bar{v}_{z}$ and an increased temperature $T$ of the vapor phase by an energy transfer between the kinetic energy of the directed and the thermal (random) motion of the particle collective.

\section{CONCLUSIONS}

A method for the study of evaporation under stationary conditions by molecular dynamics was presented. Stationarity was achieved with the introduction of reservoirs for liquid and vapor in conjunction with control volumes to specify the thermodynamic properties at the system boundaries. It allowed for sampling large molecular ensembles over an arbitrary time period of a stationary process so that statistically sound data were obtained. Typically, $N \approx 1.5 \times 10^{6}$ particles were considered. The method was validated by comparing the results of the quasistationary simulation method. Because the present approach entirely avoids searching for appropriate insertion positions, evaporation processes with dense liquids or even solids as well as high evaporation rates can be studied without difficulty.

As an independently specified physical property governing the magnitude of evaporation, the hydrodynamic velocity was selected. Large molecular ensembles allowed to approach the Euler regime, 
where true nonequilibrium phenomena, like the spread between the longitudinal and transversal temperature, vanish. In this way, the entire Knudsen layer between the interface and the Euler regime was captured. The analysis of the simulation results showed that the extent of the Knudsen layer becomes larger with a lower vapor density because of the larger mean free path and hence fewer particle interactions. A larger extent of the Knudsen layer was accompanied by a more pronounced temperature spread $T_{x y}-T_{z}$. True nonequilibrium phenomena thus become more relevant for low temperatures of the liquid and high evaporation rates that are associated with dilute vapor phases.

Varying the hydrodynamic velocity from zero, corresponding to vapor-liquid equilibrium conditions, to the speed of sound for the vacuum boundary condition, the evaporation of the Lennard-Jones truncated and shifted model fluid ${ }^{5}$ was investigated over the entire driving force range with the MD code $l s 1$ mardyn. ${ }^{32}$ The present method allows for the specification of small gradients so that realistic evaporation processes can be studied. Classical profiles of the force, temperature, density, hydrodynamic velocity, and evaporation flux were discussed, distinguishing between forward and backward flux particles. This approach led to a detailed physical insight into the involved molecular mechanisms.

\section{SUPPLEMENTARY MATERIAL}

The supplementary material states the reduction of physical properties presented in this work. It provides computational details of the conducted simulation study and validation of the present method by comparing to simulation results of conventional approaches. Moreover, the time evolution of the profiles shown in Fig. 3, covering the transition from VLE toward stationary evaporation into vacuum conditions, is depicted by a sequence of figures.

\section{ACKNOWLEDGMENTS}

This work contributes to the Collaborative Research Center SFB-TRR 75 of Deutsche Forschungsgemeinschaft (DFG) and was funded under Grant No. VR 6/9-2. All computations were performed either on the HPC cluster OCuLUS at the Paderborn Center for Parallel Computing $\left(\mathrm{PC}^{2}\right)$ or on the Cray XC40 system Hazel Hen at the High Performance Computing Center Stuttgart (HLRS) with resources allocated according to Grant No. MMHBF2. This work was carried out under the auspices of the Boltzmann-Zuse society.

\section{REFERENCES}

${ }^{1}$ H. Hertz, Ann. Phys. 253, 177 (1882).

${ }^{2}$ M. Knudsen, Ann. Phys. 352, 697 (1915).

${ }^{3}$ A. H. Persad and C. A. Ward, Chem. Rev. 116, 7727 (2016).

${ }^{4}$ Y. B. Zudin, Non-Equilibrium Evaporation and Condensation Processes (Springer, Cham, 2018).

${ }^{5}$ J. Vrabec, G. K. Kedia, G. Fuchs, and H. Hasse, Mol. Phys. 104, 1509 (2006).

${ }^{6}$ M. Heinen, J. Vrabec, and J. Fischer, J. Chem. Phys. 145, 081101 (2016).

${ }^{7}$ A. Lotfi, Ph.D. thesis, Ruhr-Universität-Bochum, Bochum, Germany, 1993.

${ }^{8}$ A. Lotfi, J. Vrabec, and J. Fischer, Int. J. Heat Mass Transfer 73, 303 (2014).

${ }^{9}$ S. I. Anisimov, D. O. Dunikov, V. V. Zhakhovskii, and S. P. Malyshenko, J. Chem. Phys. 110, 8722 (1999).

${ }^{10}$ T. Ishiyama, T. Yano, and S. Fujikawa, Phys. Fluids 16, 2899 (2004).

${ }^{11}$ A. Frezzotti, L. Gibelli, and S. Lorenzani, Phys. Fluids 17, 012102 (2005).

${ }^{12}$ A. Frezzotti, Phys. Fluids 23, 030609 (2011).

${ }^{13}$ C. Hirsch, Numerical Computation of Internal and External Flows: The Fundamentals of Computational Fluid Dynamics, 2nd ed. (Butterworth-Heinemann, Oxford, 2007).

${ }^{14}$ A. V. Gusarov and I. Smurov, Phys. Fluids 14, 4242 (2002).

${ }^{15}$ A. A. Avdeev and Y. B. Zudin, High Temp. 50, 527 (2012).

${ }^{16}$ R. Hołyst, M. Litniewski, and D. Jakubczyk, Soft Matter 13, 5858 (2017).

${ }^{17}$ Y. Tang, G. S. Grest, and S. Cheng, Langmuir 34, 7161 (2018).

${ }^{18}$ A. Frezzotti and P. Barbante, Mech. Eng. Rev. 4, 16 (2017).

${ }^{19}$ T. Tsuruta, H. Tanaka, and T. Masuoka, Int. J. Heat Mass Transfer 42, 4107 (1999).

${ }^{20}$ T. Tsuruta and G. Nagayama, Microscale Thermophys. Eng. 6, 267 (2003).

${ }^{21}$ G. Nagayama, M. Takematsu, H. Mizuguchi, and T. Tsuruta, J. Chem. Phys. 143, 014706 (2015).

${ }^{22}$ M. Bond and H. Struchtrup, Phys. Rev. E 70, 061605 (2004).

${ }^{23}$ S. Cheng, J. B. Lechman, S. J. Plimpton, and G. S. Grest, J. Chem. Phys. 134, 224704 (2011).

${ }^{24}$ R. Meland, A. Frezzotti, T. Ytrehus, and B. Hafskjold, Phys. Fluids 16, 223 (2004).

${ }^{25}$ S. Hardt and F. Wondra, J. Comput. Phys. 227, 5871 (2008).

${ }^{26}$ R. Delgado-Buscalioni and P. V. Coveney, J. Chem. Phys. 119, 978 (2003).

${ }^{27}$ J. Zhang, F. Müller-Plathe, M. Yahia-Ouahmed, and F. Leroy, J. Chem. Phys. 139, 134701 (2013).

${ }^{28}$ S. Grottel, M. Krone, C. Müller, G. Reina, and T. Ertl, IEEE Trans. Visualization Comput. Graphics 21, 201 (2015).

${ }^{29}$ T. Tsuruta and G. Nagayama, Energy 30, 795 (2005).

${ }^{30}$ T. Ishiyama, S. Fujikawa, T. Kurz, and W. Lauterborn, Phys. Rev. E 88, 042406 (2013).

${ }^{31}$ W. Press, S. Teukolsky, W. Vettering, and B. Flannery, Numerical Recipes in $\mathrm{C}++$, 2nd ed. (Cambridge University Press, Cambridge, 2002).

${ }^{32}$ C. Niethammer, S. Becker, M. Bernreuther, M. Buchholz, W. Eckhardt, A. Heinecke, S. Werth, H.-J. Bungartz, C. Glass, H. Hasse, J. Vrabec, and M. Horsch, J. Chem. Theory Comput. 10, 4455 (2014). 